\title{
On the cohomology of generalized triangle groups
}

\author{
Graham Ellis and Gerald Williams*
}

\begin{abstract}
We describe a general approach to constructing small free $\mathbb{Z} \Gamma$-resolutions for certain infinite isometry groups $\Gamma$. We apply the method to a class of generalized triangle groups and use the resolution to compute the integral homology of these groups. In illustrating the method we also obtain resolutions for the classical triangle groups and for their infinite cyclic central extensions, considered previously by Strebel.
\end{abstract}

Mathematics Subject Classification (2000). 20J06, 30F40, 57M60.

Keywords. Cohomology, resolution, triangle group, generalized triangle group.

\section{Introduction}

It was explained in [4] how, for an arbitrary finite group $H$, one can construct a small free $\mathbb{Z} H$-resolution of $\mathbb{Z}$ by first choosing a faithful linear representation $\alpha: H \rightarrow$ $\operatorname{GL}\left(\mathbb{R}^{n}\right)$ and then considering the cellular chain complex of the convex hull of the orbit of a suitable point $v \in \mathbb{R}^{n}$. Our present aim is to show that the method can also be applied to infinite groups $\Gamma$ for which one has an appropriate representation as a group of isometries of some suitable space $X$. For such groups our first approximation to a free resolution is the cellular chain complex of $X$ corresponding to a tessellation arising from the action of $\Gamma$.

As an application, we calculate resolutions for most of the groups defined by the following presentation:

$$
G(l, m, n)=\left\langle x, y \mid x^{l}=y^{m}=[x, y]^{n}=1\right\rangle,
$$

where $[x, y]=x y x^{-1} y^{-1}$ and, as throughout this paper, $l, m, n$ denote integers with $|l|,|m|,|n| \geq 2$. The groups $G(l, m, n)$ have a long history, dating back to Coxeter and Sinkov [3], [18]. More recently they have been of interest in the context of generalized triangle groups [5], [9], [10], [14], [16]. (A generalized triangle

*This work was carried out when the second author was working at NUI Galway, supported by Marie Curie fellowship HPMD-CT-2001-00079. 
group is a group that can be presented in the form $\left\langle x, y \mid x^{l}=y^{m}=w(x, y)^{n}=1\right\rangle$, where $w(x, y)$ is an element of the free product $\left\langle x, y \mid x^{l}=y^{m}=1\right\rangle$.) Generalized triangle groups have been studied for a variety of algebraic, geometric and topological reasons [1], [5], [6], [9], [10], [13], [15], [20]; Euler characteristics have been calculated [7], [23], but the cohomology of generalized triangle groups has not yet been considered. Our results can therefore be regarded as a first step towards such investigations.

This paper is structured as follows. In Section 2 we describe the method we use to obtain the resolutions. In Section 5 we use the method to provide a free $\mathbb{Z} G$-resolution for the generalized triangle group $G=G(l, m, n)$ (with some restrictions on $l, m, n$ ), from which we calculate the integral homology. In the construction of this resolution we will require resolutions for the classical triangle groups

$$
T(l, m, n)=\left\langle a, b \mid a^{l}=b^{m}=\left(a b^{-1}\right)^{n}=1\right\rangle .
$$

For this reason, in Section 3 we use the triangle groups to illustrate our method and to provide the necessary resolutions. As a slight digression, in Section 4 we also construct a resolution for the following cyclic central extensions of the classical triangle groups, whenever these are infinite:

$$
S(l, m, n)=\left\langle a, b \mid a^{l}=b^{m}=\left(a b^{-1}\right)^{n}\right\rangle .
$$

A different resolution for these extensions has previously been derived by Strebel [19] using alternative methods.

\section{General method}

Let $Y$ denote either euclidean space $E^{n}$ or hyperbolic space $H^{n}$, and let $\Gamma$ be a group with a representation as isometries of $Y$. We shall make the following assumptions:

(1) $Y$ can be embedded into some contractible $\mathrm{CW}$-space $X$ such that the action of $\Gamma$ on $Y$ extends to a cellular action on $X$.

(2) For the stabilizer group $\Gamma_{e}$ of each cell $e$ of $X$ we have some free $\mathbb{Z} \Gamma_{e}$-resolution

$$
R_{*}^{\Gamma_{e}}: \quad \cdots \longrightarrow R_{2}^{\Gamma_{e}} \longrightarrow R_{1}^{\Gamma_{e}} \longrightarrow R_{0}^{\Gamma_{e}}
$$

of the integers.

The CW-space $X$ can often be constructed as follows. Suppose $v \in Y$ is a vector such that the orbit $v^{\Gamma}=\{g v \mid g \in \Gamma\}$ is a discrete subset of $Y$. The Dirichlet-Voronoi region centred at $v$ is the set

$$
D_{\Gamma}(v)=\{x \in Y \mid\|x-v\| \leq\|x-g v\| \text { for all } 1 \neq g \in \Gamma\} .
$$


This region is a convex polyhedron which tessellates $Y$ under the action of $\Gamma$. If the region is bounded and an intersection of only finitely many half planes then the tessellation induces a CW-structure on $Y$ and we set $X=Y$. If the region is unbounded but still an intersection of only finitely many half planes, then the tessellation induces a CW-structure on a contractible space $X$ formed by suitably adjoining a discrete set of points to $Y$.

The cellular chain complex $C_{*}(X)$ is our first approximation to a free $\mathbb{Z} \Gamma$-resolution of $\mathbb{Z}$. The action of $\Gamma$ on $X$ induces an action of $\Gamma$ on $C_{*}(X)$. Since $X$ is contractible the chain complex $C_{*}(X)$ is certainly a $\mathbb{Z} \Gamma$-resolution of $\mathbb{Z}$, but in general it is not free. However, by adapting a technique of Wall [21], the resolutions $R^{\Gamma_{e}}$ can be combined with $C_{*}(X)$ to obtain a free $\mathbb{Z} \Gamma$-resolution of the integers, as we now describe.

Let $[e]$ denote the orbit of a cell $e$ in $X$ under the action of $\Gamma$, and let $\operatorname{Orb}(k)$ denote the orbits of the $k$-cells. The module $C_{p}(X)$ is a direct sum of $\mathbb{Z} \Gamma$-modules

$$
C_{p}(X)=\bigoplus_{[e] \in \operatorname{Orb}(p)}\left(\mathbb{Z} \Gamma \otimes_{\mathbb{Z} \Gamma_{e}} \mathbb{Z}\right)
$$

By defining

$$
F_{p, q}:=\bigoplus_{[e] \in \operatorname{Orb}(p)}\left(\mathbb{Z} \Gamma \otimes_{\mathbb{Z} \Gamma_{e}} R_{q}^{\Gamma_{e}}\right)
$$

we obtain a free $\mathbb{Z} \Gamma$-resolution

$$
F_{p, *}: \quad \cdots \longrightarrow F_{p, q} \longrightarrow \cdots \longrightarrow F_{p, 2} \longrightarrow F_{p, 1} \longrightarrow F_{p, 0}
$$

of the module $C_{p}(X)$.

The boundary maps in $C_{*}(X)$ induce chain maps $\partial^{h}: F_{p, q} \rightarrow F_{p-1, q}$ in the system $F_{*, *}$ of free $\mathbb{Z} \Gamma$-modules depicted in Figure 1. By construction, the 'vertical' maps $\partial^{v}: F_{p, q} \rightarrow F_{p, q-1}$ satisfy $\partial^{v} \partial^{v}=0$; however $F_{*, *}$ is not in general a bicomplex because the 'horizontal' maps $\partial^{h}$ do not necessarily square to zero. Nevertheless, we can construct a free $\mathbb{Z} \Gamma$-chain complex $R_{*}^{\Gamma}$ with $R_{n}^{\Gamma}=\bigoplus_{p+q=n} F_{p, q}$ and differential $d$ given by $\left.d\right|_{F_{p, q}}=\partial^{v}+(-1)^{p} \partial^{h}+\varepsilon$, where $\varepsilon: R_{n}^{\Gamma} \rightarrow R_{n-1}^{\Gamma}$ is a 'perturbation'. The perturbation is an infinite sum of module homomorphisms $\varepsilon=\varepsilon_{2}+\varepsilon_{3}+\cdots$ where $\varepsilon_{k}: F_{*, *} \rightarrow F_{*-k, *+k-1}$. (The maps $\varepsilon_{2}$ and $\varepsilon_{3}$ are indicated in Figure 1 by dotted and dashed arrows, respectively.) On any given summand $F_{p, q}$ only finitely many terms $\varepsilon_{k}$ are non-zero. The existence of such a perturbation follows from an easy generalization of a theorem of Wall [21] (see also [4]), but for our examples we show its existence by defining the map explicitly. The filtration on $R_{*}^{\Gamma}$ arising from the filtration by columns of $F_{*, *}$ yields a spectral sequence with $E_{p, q}^{1}=H_{q}\left(F_{p, *}\right)$. This spectral sequence implies, as in [21], that $R_{*}^{\Gamma}$ is a resolution of $\mathbb{Z}$. We shall refer to the free $\mathbb{Z} \Gamma$-resolution $R_{*}^{\Gamma}$ as the total complex of the system $F_{*, *}$. We shall use the notation $f_{i}^{p, q}$ to denote free generators of a summand $F_{p, q}$. 


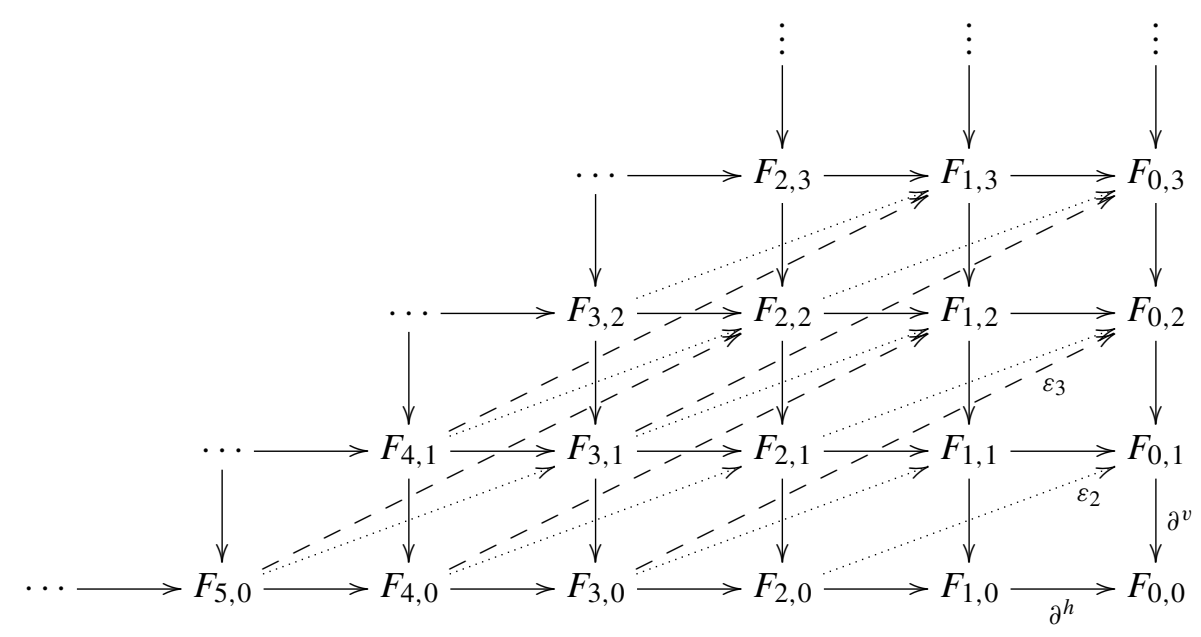

Figure 1. System $F_{*, *}$ of free $\mathbb{Z} \Gamma$-modules.

\section{Triangle groups}

We illustrate the method on the infinite triangle groups $T=T(l, m, n)$. Let $Y$ denote the hyperbolic plane $H^{2}$ if $1 /|l|+1 /|m|+1 /|n|$ is less than 1 , or the euclidean plane $E^{2}$ if $1 /|l|+1 /|m|+1 /|n|$ is equal to 1 . We define an action of $T$ on $Y$ as follows. Let $v_{1}, v_{2}$ be distinct points in $Y$, and let the generator $a$ of $T$ act as clockwise rotation about $v_{1}$ through an angle $2 \pi /|l|$, and let $b$ act as clockwise rotation about $v_{2}$ through an angle $2 \pi /|m|$. It follows that $\left(a b^{-1}\right)$ acts as anticlockwise rotation about some point $v_{3} \in Y$ by an angle $2 \pi /|n|$. Let $\triangle$ denote the triangle with vertices $v_{1}, v_{2}, v_{3}$ and let $\sigma$ denote reflection in the side $v_{1} v_{2}$. Then the region

$$
D:=\triangle \cup \sigma(\triangle)
$$

is a fundamental region for $T$. (In fact it is a Dirichlet-Voronoi region.)

Since $D$ is a finite polyhedron (a quadrilateral) the tessellation of $Y$ by $D$ under the action of $T$ yields a CW-structure, and so we set $X=Y$. The cellular action of $T$ on $X$ is summarized in Table 1 . In this table the 0 -cells $e_{1}^{0}, e_{2}^{0}, e_{3}^{0}$ are the points $v_{1}, v_{2}, v_{3}$; the boundary of a $j$-dimensional cell $e_{i}^{j}$ is an element of the (not necessarily free) $\mathbb{Z} T$-module $C_{j-1}(X)$. (We use the notation $K^{g}=g^{-1} K g$.) The cellular chain complex $C_{*}(X)$ is 2-dimensional and of the form

$$
0 \longrightarrow \mathbb{Z} T \longrightarrow \mathbb{Z} T \oplus \mathbb{Z} T \longrightarrow(\mathbb{Z} T \underset{\mathbb{Z}\langle a\rangle}{\otimes} \mathbb{Z}) \oplus(\mathbb{Z} T \underset{\mathbb{Z}\langle b\rangle}{\otimes} \mathbb{Z}) \oplus\left(\mathbb{Z} T \underset{\mathbb{Z}\left\langle a b^{-1}\right\rangle}{\otimes} \mathbb{Z}\right) .
$$


Table 1. Cellular action of $T(l, m, n)$.

\begin{tabular}{|l|l|l|l|}
\hline Cell & \multicolumn{2}{|c|}{ Boundary } & Stabilizer group \\
\hline$e^{2}$ & $\partial e^{2}$ & $\partial e^{2}=\left(1-a^{-1}\right) e_{1}^{1}+\left(1-b^{-1}\right) e_{2}^{1}$ & 1 \\
$e_{1}^{1}$ & $\partial e_{1}^{1}$ & $\partial e_{1}^{1}=-e_{1}^{0}+e_{3}^{0}$ & 1 \\
$a^{-1} e_{1}^{1}$ & $a^{-1} \partial e_{1}^{1}$ & & 1 \\
$e_{2}^{1}$ & $\partial e_{2}^{1}$ & $\partial e_{2}^{1}=e_{2}^{0}-e_{3}^{0}$ & 1 \\
$b^{-1} e_{2}^{1}$ & $b^{-1} \partial e_{2}^{1}$ & & 1 \\
$e_{1}^{0}$ & - & & $\langle a\rangle$ \\
$e_{2}^{0}$ & - & & $\langle b\rangle$ \\
$e_{3}^{0}$ & - & & $\left\langle a b^{-1}\right\rangle$ \\
$a^{-1} e_{3}^{0}$ & - & & $\left\langle a b^{-1}\right\rangle^{a}$ \\
\hline
\end{tabular}

The stabilizer groups of the 0 -cells are the cyclic groups $\langle a\rangle,\langle b\rangle,\left\langle a b^{-1}\right\rangle$, so we can use the free resolutions

$$
\begin{aligned}
& R_{*}^{\langle a\rangle}: \cdots \stackrel{\sum_{i=0}^{l-1} a^{i}}{\longrightarrow} \mathbb{Z}\langle a\rangle \stackrel{a-1}{\longrightarrow} \mathbb{Z}\langle a\rangle \stackrel{\sum_{i=0}^{l-1} a^{i}}{\longrightarrow} \mathbb{Z}\langle a\rangle \stackrel{a-1}{\longrightarrow} \mathbb{Z}\langle a\rangle, \\
& R_{*}^{\langle b\rangle}: \cdots \stackrel{\sum_{i=0}^{m-1} b^{i}}{\longrightarrow} \mathbb{Z}\langle b\rangle \stackrel{b-1}{\longrightarrow} \mathbb{Z}\langle b\rangle \stackrel{\sum_{i=0}^{m-1} b^{i}}{\longrightarrow} \mathbb{Z}\langle b\rangle \stackrel{b-1}{\longrightarrow} \mathbb{Z}\langle b\rangle, \\
& R_{*}^{\left\langle a b^{-1}\right\rangle}: \cdots \stackrel{\sum_{i=0}^{n-1}\left(a b^{-1}\right)^{i}}{\longrightarrow} \mathbb{Z}\left\langle a b^{-1}\right\rangle \stackrel{a b^{-1}-1}{\longrightarrow} \mathbb{Z}\left\langle a b^{-1}\right\rangle \\
& \stackrel{\sum_{i=0}^{n-1}\left(a b^{-1}\right)^{i}}{\longrightarrow} \mathbb{Z}\left\langle a b^{-1}\right\rangle \stackrel{a b^{-1}-1}{\longrightarrow} \mathbb{Z}\left\langle a b^{-1}\right\rangle
\end{aligned}
$$

of the integers to obtain the free resolution

$$
F_{0, *}: \quad \cdots \stackrel{\bar{\alpha}}{\longrightarrow}(\mathbb{Z} T)^{3} \stackrel{\alpha}{\longrightarrow}(\mathbb{Z} T)^{3} \stackrel{\bar{\alpha}}{\longrightarrow}(\mathbb{Z} T)^{3} \stackrel{\alpha}{\longrightarrow}(\mathbb{Z} T)^{3}
$$

of $C_{0}(X)$, where $\alpha, \bar{\alpha}$ are given in Table 2 . The stabilizers of the cells $e_{1}^{1}, e_{2}^{1}, e^{2}$ are all trivial so we immediately have the free resolutions

$$
\begin{aligned}
& F_{1, *}: 0 \longrightarrow(\mathbb{Z} T)^{2}, \\
& F_{2, *}: 0 \longrightarrow(\mathbb{Z} T),
\end{aligned}
$$

of $C_{1}(X)$ and $C_{2}(X)$ respectively. We thus obtain the system of free $\mathbb{Z} T$-modules in Figure 2, where the vertical maps $\alpha, \bar{\alpha}$, the induced horizontal maps $\delta_{2}, \delta_{1}$, and the perturbation map $\xi$ are given in Table 2. The total complex of this system is then the 
Table 2. Boundary maps for system of free $\mathbb{Z} T$-modules.

\begin{tabular}{|c|c|}
\hline Summand & Boundary map \\
\hline$F_{2,0}$ & $\begin{array}{l}\delta_{2}\left(f^{2,0}\right)=\left(a^{-1}-1\right) f_{1}^{1,0}+\left(b^{-1}-1\right) f_{2}^{1,0} \\
\xi\left(f^{2,0}\right)=a^{-1} f_{1}^{0,1}-b^{-1} f_{2}^{0,1}-a^{-1} f_{3}^{0,1}\end{array}$ \\
\hline$F_{1,0}$ & $\begin{aligned} \delta_{1}\left(f_{1}^{1,0}\right) & =f_{1}^{0,0}-f_{3}^{0,0} \\
\delta_{1}\left(f_{2}^{1,0}\right) & =-f_{2}^{0,0}+f_{3}^{0,0}\end{aligned}$ \\
\hline $\begin{array}{l}F_{0,2 q} \\
(q \geq 1)\end{array}$ & $\begin{array}{l}\bar{\alpha}\left(f_{1}^{0,2 q}\right)=\left(\sum_{i=0}^{l-1} a^{i}\right) f_{1}^{0,2 q-1} \\
\bar{\alpha}\left(f_{2}^{0,2 q}\right)=\left(\sum_{i=0}^{m-1} b^{i}\right) f_{2}^{0,2 q-1} \\
\bar{\alpha}\left(f_{3}^{0,2 q}\right)=\left(\sum_{i=0}^{n-1}\left(a b^{-1}\right)^{i}\right) f_{3}^{0,2 q-1}\end{array}$ \\
\hline $\begin{array}{l}F_{0,2 q+1} \\
(q \geq 0)\end{array}$ & $\begin{array}{l}\alpha\left(f_{1}^{0,2 q+1}\right)=(a-1) f_{1}^{0,2 q} \\
\alpha\left(f_{2}^{0,2 q+1}\right)=(b-1) f_{2}^{0,2 q} \\
\alpha\left(f_{3}^{0,2 q+1}\right)=\left(a b^{-1}-1\right) f_{3}^{0,2 q}\end{array}$ \\
\hline
\end{tabular}

following free $\mathbb{Z} T$-resolution of the integers:

$$
\begin{aligned}
\cdots \stackrel{\bar{\alpha}}{\longrightarrow} & (\mathbb{Z} T)^{3} \stackrel{\alpha}{\longrightarrow}(\mathbb{Z} T)^{3} \stackrel{\bar{\alpha}}{\longrightarrow}(\mathbb{Z} T)^{3} \stackrel{\alpha}{\longrightarrow}(\mathbb{Z} T)^{3} \\
& \stackrel{\bar{\alpha}}{\longrightarrow}(\mathbb{Z} T)^{3} \stackrel{\alpha}{\longrightarrow}(\mathbb{Z} T)^{4} \stackrel{d_{2}}{\longrightarrow}(\mathbb{Z} T)^{5} \stackrel{d_{1}}{\longrightarrow}(\mathbb{Z} T)^{3}
\end{aligned}
$$

where $d_{1}, d_{2}$ are given by

$$
\begin{aligned}
& d_{1}=\alpha+\delta_{1}, \\
& d_{2}=\bar{\alpha}+\delta_{2}+\xi
\end{aligned}
$$

The resolution can be used to make calculations. For instance, in dimensions $k \geq 3$ we have the known result that

$$
H_{k}(T, A) \cong H_{k}\left(\mathbb{Z}_{l}, A\right) \oplus H_{k}\left(\mathbb{Z}_{m}, A\right) \oplus H_{k}\left(\mathbb{Z}_{n}, A\right)
$$

for any $\mathbb{Z} T$-module $A$. Also $H_{2}(T, \mathbb{Z}) \cong \mathbb{Z}$.

Free resolutions for finite triangle groups $T$ can be obtained using the methods of [4]. In Section 5 we will require free resolutions of the integers for the group $T(3,3,2)$ (isomorphic to $T(3,2,3)$ and the alternating group $\left.A_{4}\right)$ and for the group $T(2,2, n)$ (isomorphic to the dihedral group of order $\left.|2 n|, D_{|2 n|}\right)$. We sketch the derivation of a resolution for $T=T(3,2,3)$.

Suppose $\psi: T \rightarrow A_{4}$ is an isomorphism. Assume that $g \in T$ acts on a vector $\alpha=\left(\alpha_{1}, \alpha_{2}, \alpha_{3}, \alpha_{4}\right) \in \mathbb{R}^{4}$ by

$$
g \cdot \alpha=\left(\alpha_{\psi\left(g^{-1}\right)(1)}, \alpha_{\psi\left(g^{-1}\right)(2)}, \alpha_{\psi\left(g^{-1}\right)(3)}, \alpha_{\psi\left(g^{-1}\right)(4)}\right) .
$$




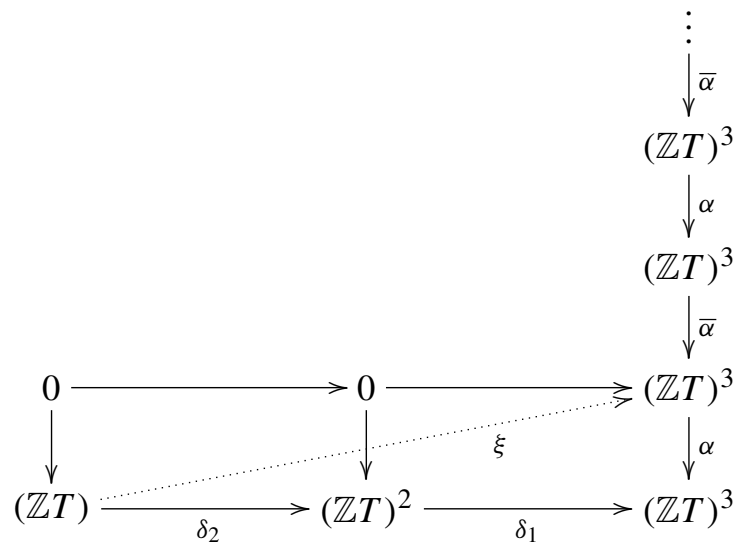

Figure 2. System of free $\mathbb{Z} T$-modules.

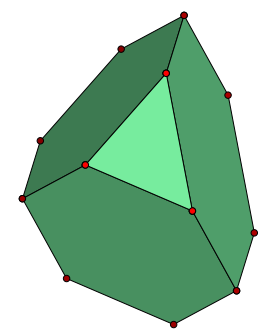

Figure 3. Polytope $P$.

Under this action the orbit of the vector $v=(1,2,3,1)$ is a collection of 12 vectors whose convex hull is the 3-dimensional polytope $P$ pictured in Figure 3 . Here the triangular faces have boundary label $a^{3}$ and the hexagonal faces have boundary label $\left(a b^{-1}\right)^{3}$. The triangles have stabilizer group $\left\langle a \mid a^{3}\right\rangle$, the hexagons have stabilizer group $\left\langle\left(a b^{-1}\right) \mid\left(a b^{-1}\right)^{3}\right\rangle$, the edges labelled $a$ have trivial stabilizer group, and the edges labelled $b$ have stabilizer group $\left\langle b \mid b^{2}\right\rangle$.

The action of $T$ on $\mathbb{R}^{4}$ induces a cellular action of $T$ on $P$. The cellular chain complex $C_{*}(P)$ can be regarded as a 3 -dimensional chain complex of $\mathbb{Z} T$-modules. Now $P$ is contractible so $H_{k}(P, \mathbb{Z})=0$ for $k \geq 1$ and $H_{0}(P, \mathbb{Z})=\mathbb{Z}$. Since $C_{3}(P)=\mathbb{Z}$ we can splice together copies of $C_{*}(P)$ to form a $\mathbb{Z} G$-resolution of the integers

$$
\begin{aligned}
C_{*}^{\prime}(P): \cdots & \longrightarrow C_{1}(P) \longrightarrow C_{0}(P) \longrightarrow C_{2}(P) \longrightarrow C_{1}(P) \\
& \longrightarrow C_{0}(P) \longrightarrow C_{2}(P) \longrightarrow C_{1}(P) \longrightarrow C_{0}(P) .
\end{aligned}
$$


As $\mathbb{Z} T$-modules we have

$$
\begin{aligned}
& C_{0}(P)=(\mathbb{Z} T), \\
& C_{1}(P)=(\mathbb{Z} T) \oplus(\mathbb{Z} T \underset{\mathbb{Z}\langle b\rangle}{\otimes} \mathbb{Z}), \\
& C_{2}(P)=(\mathbb{Z} T \underset{\mathbb{Z}\langle a\rangle}{\otimes} \mathbb{Z}) \oplus\left(\mathbb{Z} T \underset{\mathbb{Z}\left\langle a b^{-1}\right\rangle}{\otimes} \mathbb{Z}\right) .
\end{aligned}
$$

Using Wall's perturbation technique to combine standard free resolutions for the cyclic groups $\left\langle a \mid a^{3}\right\rangle,\left\langle b \mid b^{2}\right\rangle,\left\langle\left(a b^{-1}\right) \mid\left(a b^{-1}\right)^{3}\right\rangle$ with the (non-free) resolution $C_{*}^{\prime}(P)$ we obtain a free $\mathbb{Z} T$-resolution of the integers

$$
R_{*}: \quad \cdots \longrightarrow R_{6} \longrightarrow R_{5} \longrightarrow R_{4} \longrightarrow R_{3} \longrightarrow R_{2} \longrightarrow R_{1} \longrightarrow R_{0}
$$

where for $p \geq 0, R_{p}=(\mathbb{Z} T)^{p+1}$.

The same method can be applied in the case $T \cong D_{|2 n|}$. If the standard action of $D_{|2 n|}$ on $\mathbb{R}^{2}$ is used, then the resolution obtained is again of the form (5) where for $p \geq 0, R_{p}=(\mathbb{Z} T)^{p+1}$.

\section{Central extensions of triangle groups}

We now turn our attention to the groups $S=S(l, m, n)$ defined in (3). The element $\left(a b^{-1}\right)^{n}$ of $S(l, m, n)$ is a power of each generator, so is central. Since $S(l, m, n) /\left\langle\left(a b^{-1}\right)^{n}\right\rangle \cong T(l, m, n)$ we have that the groups $S(l, m, n)$ are cyclic central extensions of the triangle groups $T(l, m, n)$.

The group $T(l, m, n)$ is infinite if and only if $1 /|l|+1 /|m|+1 /|n| \leq 1$. Since $S(l, m, n)$ maps homomorphically onto $T(l, m, n)$, if $1 /|l|+1 /|m|+1 /|n| \leq 1$ then $S(l, m, n)$ is infinite. For the converse, suppose that $1 /|l|+1 /|m|+1 /|n|>1$, i.e. $\{|l|,|m|,|n|\}=\{3,3,2\},\{3,4,2\},\{3,5,2\}$ or $\{2,2, q\}$ where $q \geq 2$. In the first three cases, calculations in GAP [8] show that $S(l, m, n)$ is finite. In the case $\{|l|,|m|,|n|\}=\{2,2, q\}$, the group $S(l, m, n)$ is isomorphic to one of $S(q, 2,2)$, $S(q,-2,2), S(2,-q, 2), S(-2,-q, 2)$. It is easy to show that the abelianizations of these groups are finite of orders $4,4(q-1), 4,4(q+1)$, respectively. The central extension $\left\langle\left(a b^{-1}\right)^{n}\right\rangle \triangleleft S(l, m, n) \rightarrow T(l, m, n)$ yields the exact sequence

$$
H_{2}(S, \mathbb{Z}) \longrightarrow H_{2}(T, \mathbb{Z}) \longrightarrow\left\langle\left(a b^{-1}\right)^{n}\right\rangle \longrightarrow S^{\mathrm{ab}} \longrightarrow T^{\mathrm{ab}} \longrightarrow 0 .
$$

Since $T$ is finite so is $H_{2}(T, \mathbb{Z})$, and (6) then shows that $\left\langle\left(a b^{-1}\right)^{n}\right\rangle$, and hence $S$, is finite. Thus we have shown that $S(l, m, n)$ is infinite if and only if $1 /|l|+1 /|m|+$ $1 /|n| \leq 1$. For a more detailed analysis of the finite groups $S(l, m, n)$ see [19].

We will calculate a free $\mathbb{Z} S$-resolution of the integers whenever $S$ is infinite. A different resolution was obtained in [19] using alternative methods. We will require the following technical result. 
Lemma 1. If $S=S(l, m, n)$ is infinite then the subgroup $\left\langle\left(a b^{-1}\right)^{n}\right\rangle$ is infinite.

Proof. If $1 / l-1 / m=1 / n$ then there exists a representation $S(l, m, n) \rightarrow \mathbb{Z}$ given by $a \mapsto m n, b \mapsto l n$. Under this map the element $\left(a b^{-1}\right)^{n}$ of $S(l, m, n)$ maps to $l m n \in \mathbb{Z}$. This is of infinite order, so the subgroup $\left\langle\left(a b^{-1}\right)^{n}\right\rangle$ of $S(l, m, n)$ is infinite, as required. Assume then that $1 / l-1 / m \neq 1 / n$.

Let $R_{1}=\left(a b^{-1}\right)^{n} a^{-l}, R_{2}=\left(a b^{-1}\right)^{n} b^{-m}$. As explained in [2], [17] the presentation $S(l, m, n)=\left\langle a, b \mid R_{1}, R_{2}\right\rangle$ yields a resolution

$$
\cdots \longrightarrow C_{4} \longrightarrow C_{3} \longrightarrow(\mathbb{Z} S)^{2} \stackrel{\delta_{2}}{\longrightarrow}(\mathbb{Z} S)^{2} \longrightarrow(\mathbb{Z} S)
$$

of the integers. The map $\delta_{2}$ is given by

$$
\begin{aligned}
& \delta_{2}: e_{1}^{2} \mapsto \frac{\partial R_{1}}{\partial a} e_{1}^{1}+\frac{\partial R_{1}}{\partial b} e_{2}^{2}, \\
& \delta_{2}: e_{2}^{2} \mapsto \frac{\partial R_{2}}{\partial a} e_{1}^{1}+\frac{\partial R_{2}}{\partial b} e_{2}^{2},
\end{aligned}
$$

where $\partial / \partial a, \partial / \partial b$ denote Fox derivatives with respect to $a$ and $b$, and where $e_{1}^{2}, e_{2}^{2}$ are the free generators of $C_{2}$, and $e_{1}^{1}, e_{2}^{1}$ are the free generators of $C_{1}$. Tensoring with $\mathbb{Z}$ over $\mathbb{Z} S$ yields a chain complex

$$
\cdots \longrightarrow C_{4} \underset{\mathbb{Z} S}{\otimes} \mathbb{Z} \longrightarrow C_{3} \underset{\mathbb{Z} S}{\otimes} \mathbb{Z} \longrightarrow \mathbb{Z}^{2} \stackrel{\delta_{2} \otimes_{\mathbb{Z} S} \mathbb{Z}}{\longrightarrow} \mathbb{Z}^{2} \longrightarrow \mathbb{Z}
$$

The required Fox derivatives are given by

$$
\begin{aligned}
\partial R_{1} / \partial a= & \operatorname{sign}(n)\left(a b^{-1}\right)^{(n-|n|) / 2}\left(1+\left(a b^{-1}\right)+\cdots+\left(a b^{-1}\right)^{|n|-1}\right) \\
& -\operatorname{sign}(l)\left(a b^{-1}\right)^{n} a^{-(l+|l|) / 2}\left(1+a+\cdots+a^{|l|-1}\right), \\
\partial R_{1} / \partial b= & \operatorname{sign}(n)\left(a b^{-1}\right)^{(n-|n|) / 2}\left(1+\left(a b^{-1}\right)+\cdots+\left(a b^{-1}\right)^{|n|-1}\right)\left(-a b^{-1}\right), \\
\partial R_{2} / \partial a= & \operatorname{sign}(n)\left(a b^{-1}\right)^{(n-|n|) / 2}\left(1+\left(a b^{-1}\right)+\cdots+\left(a b^{-1}\right)^{|n|-1}\right), \\
\partial R_{2} / \partial b= & \operatorname{sign}(n)\left(a b^{-1}\right)^{(n-|n|) / 2}\left(1+\left(a b^{-1}\right)+\cdots+\left(a b^{-1}\right)^{|n|-1}\right)\left(-a b^{-1}\right) \\
& -\operatorname{sign}(m)\left(a b^{-1}\right)^{n} b^{-(m+|m|) / 2}\left(1+b+\cdots+b^{|m|-1}\right),
\end{aligned}
$$

so the map $\delta_{2} \otimes_{\mathbb{Z} S} \mathbb{Z}$ is given by

$$
\begin{aligned}
& \delta_{2} \otimes_{\mathbb{Z} S} \mathbb{Z}: \bar{e}_{1}^{2} \mapsto(n-l) \bar{e}_{1}^{1}-n \bar{e}_{2}^{2}, \\
& \delta_{2} \otimes_{\mathbb{Z} S} \mathbb{Z}: \bar{e}_{2}^{2} \mapsto n \bar{e}_{1}^{1}-(n+m) \bar{e}_{2}^{2} .
\end{aligned}
$$

Since $1 / l-1 / m \neq 1 / n$ this is an injective map, and $H_{2}(S, \mathbb{Z})=0$. The five term exact sequence (6) now implies that $H_{2}(T, \mathbb{Z})$ injects into $\left\langle\left(a b^{-1}\right)^{n}\right\rangle$. Using the resolution obtained in Section 3 we have that $H_{2}(T, \mathbb{Z}) \cong \mathbb{Z}$ and thus $\left\langle\left(a b^{-1}\right)^{n}\right\rangle$ is infinite. 
Theorem 2. Let $S=S(l, m, n)$ where $1 /|l|+1 /|m|+1 /|n| \leq 1$. Then there is a free 3-dimensional $\mathbb{Z} S$-resolution of the integers

$$
0 \longrightarrow(\mathbb{Z} S) \longrightarrow(\mathbb{Z} S)^{3} \longrightarrow(\mathbb{Z} S)^{5} \longrightarrow(\mathbb{Z} S)^{3}
$$

obtained as the total complex of the system of free $\mathbb{Z} S$-modules in Figure 4.

Proof. An action of $S$ on the euclidean or hyperbolic plane $Y$ can be defined in the same way as the action of $T$ on $Y$ was defined in Section 3. As before, this yields a CW-structure so we set $X=Y$. The cellular action of $S$ on $X$ is the same as the action of $T$ on $X$ (summarized in Table 1), except that the stabilizer group of 2-cell $e^{2}$ is the group $\left\langle a^{l}\right\rangle$, and the stabilizer groups of the 1 -cells $e_{1}^{1}, e_{2}^{1}$ are the groups $\left\langle a^{l}\right\rangle,\left\langle b^{m}\right\rangle$, respectively. (Note that since the 2 and 3-dimensional cells have nontrivial stabilizers the action of $S$ is not faithful.) The cellular chain complex $C_{*}(X)$ is 2-dimensional of the form

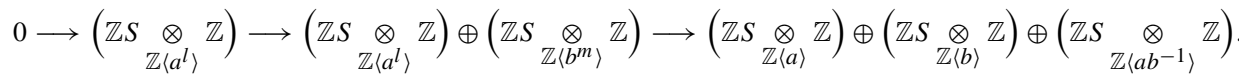

By Lemma 1 each of the stabilizer groups $S_{e}$ is isomorphic to the infinite cyclic group, so for each cell $e \in C_{*}(X)$ we can use resolutions $R_{*}^{S_{e}}$ of the form

$$
R_{*}^{S_{e}}: 0 \longrightarrow \mathbb{Z} S_{e} \longrightarrow \mathbb{Z} S_{e} .
$$

We then have the free resolutions

$$
\begin{array}{ll}
F_{0, *}: & 0 \longrightarrow(\mathbb{Z} S)^{3} \stackrel{\alpha}{\longrightarrow}(\mathbb{Z} S)^{3}, \\
F_{1, *}: & 0 \longrightarrow(\mathbb{Z} S)^{2} \stackrel{\beta}{\longrightarrow}(\mathbb{Z} S)^{2}, \\
F_{2, *}: & 0 \longrightarrow(\mathbb{Z} S) \stackrel{\gamma}{\longrightarrow}(\mathbb{Z} S),
\end{array}
$$

of $C_{0}(X), C_{1}(X), C_{2}(X)$ respectively, where $\alpha, \beta, \gamma$ are given in Table 3 . We thus obtain the system of free $\mathbb{Z} S$-modules in Figure 4, where the vertical maps $\alpha, \beta, \gamma$, the induced horizontal maps $\delta_{1}, \bar{\delta}_{1}, \delta_{2}$, and the pertubation map $\xi$ are given in Table 3 . (Note that the maps are defined in such a way that the squares in this system anti-

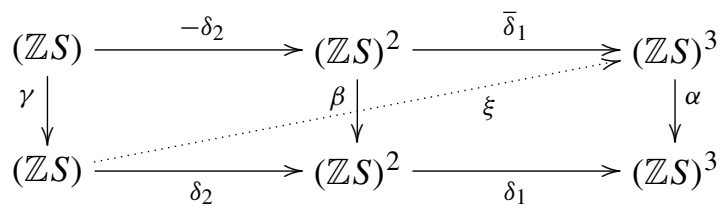

Figure 4. System of free $\mathbb{Z} S$-modules. 
Table 3. Boundary maps for system of free $\mathbb{Z} S$-modules.

\begin{tabular}{|l|l|}
\hline Summand & Boundary map \\
\hline$F_{2, q}$ & $\delta_{2}\left(f^{2, q}\right)=\left(a^{-1}-1\right) f_{1}^{1, q}+\left(b^{-1}-1\right) f_{2}^{1, q}$ \\
\hline$F_{2,1}$ & $\gamma\left(f^{2,1}\right)=\left(a^{l}-1\right) f_{1}^{2,0}$ \\
\hline$F_{2,0}$ & $\xi\left(f_{1}^{2,0}\right)=a^{-1} f_{1}^{0,1}-b^{-1} f_{2}^{0,1}-a^{-1} f_{3}^{0,1}$ \\
\hline$F_{1,1}$ & $\beta\left(f_{1}^{1,1}\right)=\left(a^{l}-1\right) f_{1}^{1,0}$ \\
& $\beta\left(f_{2}^{1,1}\right)=\left(b^{m}-1\right) f_{2}^{1,0}$ \\
& $\bar{\delta}_{1}\left(f_{1}^{1,1}\right)=-\left(\sum_{i=0}^{l-1} a^{i}\right) f_{1}^{0,1}+\left(\sum_{i=0}^{n-1}\left(a b^{-1}\right)^{i}\right) f_{3}^{0,1}$ \\
& $\bar{\delta}_{1}\left(f_{2}^{1,1}\right)=\left(\sum_{i=0}^{m-1} b^{i}\right) f_{2}^{0,1}-\left(\sum_{i=0}^{n-1}\left(a b^{-1}\right)^{i}\right) f_{3}^{0,1}$ \\
& $\delta_{1}\left(f_{1}^{1,0}\right)=f_{1}^{0,0}-f_{3}^{0,0}$ \\
$F_{1,0}\left(f_{2}^{1,0}\right)=-f_{2}^{0,0}+f_{3}^{0,0}$ & $\alpha\left(f_{1}^{0,1}\right)=(a-1) f_{1}^{0,0}$ \\
& $\alpha\left(f_{2}^{0,1}\right)=(b-1) f_{2}^{0,0}$ \\
& $\alpha\left(f_{3}^{0,1}\right)=\left(a b^{-1}-1\right) f_{3}^{0,0}$ \\
\hline$F_{0,1}$ &
\end{tabular}

commute.) The total complex of this system is then the following free $\mathbb{Z} S$-resolution of the integers:

$$
0 \longrightarrow(\mathbb{Z} S) \stackrel{d_{3}}{\longrightarrow}(\mathbb{Z} S)^{3} \stackrel{d_{2}}{\longrightarrow}(\mathbb{Z} S)^{5} \stackrel{d_{1}}{\longrightarrow}(\mathbb{Z} S)^{3}
$$

where $d_{1}, d_{2}, d_{3}$ are given by

$$
\begin{aligned}
& d_{1}=\alpha+\delta_{1}, \\
& d_{2}=\bar{\delta}_{1}+\beta+\delta_{2}+\xi, \\
& d_{3}=-\delta_{2}+\gamma .
\end{aligned}
$$

This resolution can be used to make calculations such as the following. If $A$ is any $\mathbb{Z} S$-module then $H_{k}(S, A)=0$ when $k \geq 4$. Also $H_{3}(S, \mathbb{Z})=\mathbb{Z} ; H_{2}(S, \mathbb{Z})=\mathbb{Z}$ if $1 / l-1 / m=1 / n$ and $H_{2}(S, \mathbb{Z})=0$ otherwise. 


\section{Generalized triangle groups}

We now consider the groups $G=G(l, m, n)$. If $\{|l|,|m|\}=\{2,2\}$ then $G \cong D_{|4 n|}$; if $(\{|l|,|m|\},|n|)=(\{2,3\}, 2)$ then $G \cong A_{4} \times \mathbb{Z}_{2}$; if $(\{|l|,|m|\},|n|)=(\{3,3\}, 2)$ then $G$ is finite of order 288 ; if $(\{|l|,|m|\},|n|)=(\{2,4\}, 2)$ or $(\{2,3\}, 3)$ then $G$ is infinite and soluble [14]. In all other cases there exists a faithful action of $G$ on hyperbolic 3-space $H^{3}$ [9], [10]. The Euler characteristics of generalized triangle groups admitting such an action were calculated in [23] and we have

$$
\chi(G)=\frac{\min \{0,2 /|l|+1 /|n|-1\}+\min \{0,2 /|m|+1 /|n|-1\}}{2} .
$$

The orbifold corresponding to the faithful representation of $G$ is non-compact in every case, and it has finite volume precisely in the cases $(\{3,3\}, 3),(\{3,4\}, 2)$, $(\{4,4\}, 2)$. In these cases $G$ has a faithful representation as an arithmetic Kleinian group; the $(\{4,4\}, 2)$ and $(\{3,4\}, 2)$ groups are commensurable with the Picard group PSL $\left(2, \mathcal{O}_{1}\right)$ and the $(\{3,3\}, 3)$ group is commensurable with the Bianchi group $\operatorname{PSL}\left(2, \mathcal{O}_{3}\right)$ (where $\mathcal{O}_{d}$ denotes the ring of integers in $\mathbb{Q}(\sqrt{-d})$ ) [10].

We apply our method to obtain free $\mathbb{Z} G$-resolutions of the integers in all cases where there is a faithful action on $H^{3}$.

Theorem 3. Let $G=G(l, m, n)$ where $2 /|l|+1 /|n| \leq 1$ and $2 /|m|+1 /|n| \leq 1$. Then there is a free $\mathbb{Z} G$-resolution of the integers

$$
\begin{aligned}
\cdots \longrightarrow & (\mathbb{Z} G)^{9} \longrightarrow(\mathbb{Z} G)^{9} \longrightarrow(\mathbb{Z} G)^{9} \\
& \longrightarrow(\mathbb{Z} G)^{10} \longrightarrow(\mathbb{Z} G)^{13} \longrightarrow(\mathbb{Z} G)^{13} \longrightarrow(\mathbb{Z} G)^{6}
\end{aligned}
$$

obtained as the total complex of the system of free $\mathbb{Z} G$-modules in Figure 5.

Proof. Let $Y$ denote hyperbolic 3-space $H^{3}$. It was shown in [9], [10] that there exists a faithful action of $G$ on $Y$, which we now describe. There exist skew axes $L_{1}, L_{2}$ in $Y$ such that $x$ acts as rotation through an angle $2 \pi / l$ about $L_{1}$ and $y$ acts as rotation through an angle $2 \pi / m$ about $L_{2}$. The triangle subgroups $\left\langle x, y x y^{-1}\right\rangle,\left\langle x, y x y^{-1}\right\rangle^{y}$ of $G$ (isomorphic to $T(l, l, n)$ ) act on $Y$ with 'fixed points' $v_{1}, y^{-1} v_{1}$, respectively. Using the language of [20], $v_{1}, y^{-1} v_{1}$ are ordinary points in $H^{3}$, infinite points on the boundary $\partial H^{3}$, or ideal points outside $H^{3}$ depending on whether the value of $2 /|l|+$ $1 /|n|-1$ is positive, zero, or negative, respectively. Similarly the triangle subgroups $\left\langle y, x y x^{-1}\right\rangle,\left\langle y, x y x^{-1}\right\rangle^{x}$ of $G$ (isomorphic to $\left.T(m, m, n)\right)$ act on $Y$ with fixed points $v_{2}, x^{-1} v_{2}$ respectively. These points are ordinary, infinite, or ideal depending on the value of $2 /|m|+1 /|n|-1$. The four points $v_{1}, y^{-1} v_{1}, v_{2}, x^{-1} v_{2}$ form a 'tetrahedron' in $Y$; this tetrahedron is a fundamental domain for $G$.

Under the hypotheses of the theorem $v_{1}, v_{2}$ are actually either infinite or ideal points. We form the contractible space $X$ by adjoining 0-cells to $Y$ in one to one 
correspondence with points in the orbits of $v_{1}, v_{2}$. The tessellation of $Y$ under the action of $G$ induces a CW-structure on $X$. The cellular action of $G$ on $X$ is summarized in Table 4 . In this table the 0 -cells $e_{1}^{0}, e_{2}^{0}$ correspond to the points $v_{1}, v_{2}$; the boundary

Table 4. Cellular action of $G(l, m, n)$.

\begin{tabular}{|l|l|l|l|}
\hline Cell & \multicolumn{2}{|c|}{ Boundary } & Stabilizer group \\
\hline$e^{3}$ & $\partial e^{3}$ & $\partial e^{3}=\left(1-x^{-1}\right) e_{1}^{2}+\left(1-y^{-1}\right) e_{2}^{2}$ & 1 \\
$e_{1}^{2}$ & $\partial e_{1}^{2}$ & $\partial e_{1}^{2}=e_{1}^{1}+\left(y^{-1}-1\right) e_{3}^{1}$ & 1 \\
$x^{-1} e_{1}^{2}$ & $x^{-1} \partial e_{1}^{2}$ & & 1 \\
$e_{2}^{2}$ & $\partial e_{2}^{2}$ & $\partial e_{2}^{2}=e_{2}^{1}+\left(1-x^{-1}\right) e_{3}^{1}$ & 1 \\
$y^{-1} e_{2}^{2}$ & $y^{-1} \partial e_{2}^{2}$ & & 1 \\
$e_{1}^{1}$ & $\partial e_{1}^{1}$ & $\partial e_{1}^{1}=\left(1-y^{-1}\right) e_{1}^{0}$ & $\langle x\rangle$ \\
$e_{2}^{1}$ & $\partial e_{2}^{1}$ & $\partial e_{2}^{1}=\left(1-x^{-1}\right) e_{2}^{0}$ & $\langle y\rangle$ \\
$e_{3}^{1}$ & $\partial e_{3}^{1}$ & $\partial e_{3}^{1}=e_{1}^{0}-e_{2}^{0}$ & $\langle[x, y]\rangle$ \\
$y^{-1} e_{3}^{1}$ & $y^{-1} \partial e_{3}^{1}$ & & $\langle[x, y]\rangle^{y}$ \\
$x^{-1} y^{-1} e_{3}^{1}$ & $x^{-1} y^{-1} \partial e_{3}^{1}$ & & $\langle[x, y]\rangle^{y x}$ \\
$x^{-1} e_{3}^{1}$ & $x^{-1} \partial e_{3}^{1}$ & & $\langle[x, y]\rangle^{x}$ \\
$e_{1}^{0}$ & - & & $\left\langle x, y x y^{-1}\right\rangle$ \\
$y^{-1} e_{1}^{0}$ & - & & $\left\langle x, y x y^{-1}\right\rangle^{y}$ \\
$e_{2}^{0}$ & - & & $\left\langle y, x y x^{-1}\right\rangle$ \\
$x^{-1} e_{2}^{0}$ & - & & $\left\langle y, x y x^{-1}\right\rangle^{x}$ \\
\hline
\end{tabular}

of a $j$-dimensional cell $e_{i}^{j}$ is an element of the (not necessarily free) $\mathbb{Z} G$-module $C_{j-1}(X)$. The (3-dimensional) cellular chain complex $C_{*}(X)$ is of the form

$$
0 \longrightarrow C_{3}(X) \longrightarrow C_{2}(X) \longrightarrow C_{1}(X) \longrightarrow C_{0}(X)
$$

where

$$
\begin{aligned}
& C_{3}(X)=(\mathbb{Z} G), \\
& C_{2}(X)=(\mathbb{Z} G)^{2}, \\
& C_{1}(X)=(\mathbb{Z} G \underset{\mathbb{Z}\langle x\rangle}{\otimes} \mathbb{Z}) \oplus(\mathbb{Z} G \underset{\mathbb{Z}\langle y\rangle}{\otimes} \mathbb{Z}) \oplus(\mathbb{Z} G \underset{\mathbb{Z}\langle[x, y]\rangle}{\otimes} \mathbb{Z}), \\
& C_{0}(X)=\left(\mathbb{Z} G \underset{\mathbb{Z}\left\langle x, y x y^{-1}\right\rangle}{\otimes} \mathbb{Z}\right) \oplus\left(\mathbb{Z} G \underset{\mathbb{Z}\left\langle y, x y x^{-1}\right\rangle}{\otimes} \mathbb{Z}\right) .
\end{aligned}
$$


The stabilizer groups of the 0-cells are triangle groups, so we can use free resolutions of the form given in Section 3 to obtain the free resolution

$$
\begin{aligned}
F_{0, *}: \quad \cdots \stackrel{\bar{\alpha}}{\longrightarrow}(\mathbb{Z} G)^{6} \stackrel{\alpha}{\longrightarrow}(\mathbb{Z} G)^{6} \\
\quad \stackrel{\bar{\alpha}}{\longrightarrow}(\mathbb{Z} G)^{6} \stackrel{\sigma}{\longrightarrow}(\mathbb{Z} G)^{8} \stackrel{\nu}{\longrightarrow}(\mathbb{Z} G)^{10} \stackrel{\mu}{\longrightarrow}(\mathbb{Z} G)^{6}
\end{aligned}
$$

of $C_{0}(X)$, where $\alpha, \bar{\alpha}, \sigma, \nu, \mu$ are given in Table 5 (see p. 586). The stabilizer groups of the 1-cells are the cyclic groups $\langle x\rangle,\langle y\rangle,\langle[x, y]\rangle$, so we can use the free resolutions

$$
\begin{aligned}
& R_{*}^{\langle x\rangle}: \quad \cdots \stackrel{\sum_{i=0}^{l-1} x^{i}}{\longrightarrow} \mathbb{Z}\langle x\rangle \stackrel{x-1}{\longrightarrow} \mathbb{Z}\langle x\rangle \stackrel{\sum_{i=0}^{l-1} x^{i}}{\longrightarrow} \mathbb{Z}\langle x\rangle \stackrel{x-1}{\longrightarrow} \mathbb{Z}\langle x\rangle, \\
& R_{*}^{\langle y\rangle}: \quad \cdots \stackrel{\sum_{i=0}^{m-1} y^{i}}{\longrightarrow} \mathbb{Z}\langle y\rangle \stackrel{y-1}{\longrightarrow} \mathbb{Z}\langle y\rangle \stackrel{\sum_{i=0}^{m-1} y^{i}}{\longrightarrow} \mathbb{Z}\langle y\rangle \stackrel{y-1}{\longrightarrow} \mathbb{Z}\langle y\rangle, \\
& R_{*}^{\langle[x, y]\rangle}: \quad \ldots \stackrel{\sum_{i=0}^{n-1}[x, y]^{i}}{\longrightarrow} \mathbb{Z}\langle[x, y]\rangle \stackrel{[x, y]-1}{\longrightarrow} \mathbb{Z}\langle[x, y]\rangle \\
& \stackrel{\sum_{i=0}^{n-1}[x, y]^{i}}{\longrightarrow} \mathbb{Z}\langle[x, y]\rangle \stackrel{[x, y]-1}{\longrightarrow} \mathbb{Z}\langle[x, y]\rangle,
\end{aligned}
$$

to obtain the free resolution

$$
F_{1, *}: \quad \cdots \stackrel{\bar{\beta}}{\longrightarrow}(\mathbb{Z} G)^{3} \stackrel{\beta}{\longrightarrow}(\mathbb{Z} G)^{3} \stackrel{\bar{\beta}}{\longrightarrow}(\mathbb{Z} G)^{3} \stackrel{\beta}{\longrightarrow}(\mathbb{Z} G)^{3}
$$

of $C_{1}(X)$, where $\beta, \bar{\beta}$ are given in Table 5. The stabilizers of the cells $e_{1}^{2}, e_{2}^{2}, e^{3}$ are all trivial so we immediately have the free resolutions

$$
\begin{aligned}
& F_{2, *}: 0 \longrightarrow(\mathbb{Z} G)^{2}, \\
& F_{3, *}: 0 \longrightarrow(\mathbb{Z} G),
\end{aligned}
$$

of $C_{2}(X)$ and $C_{3}(X)$ respectively.

We thus obtain the system of free $\mathbb{Z} G$-modules depicted in Figure 5 , where the vertical maps $\mu, v, \sigma, \alpha, \bar{\alpha}, \beta, \bar{\beta}$, the induced horizontal maps $\delta_{1}, \bar{\delta}_{1}, \tilde{\delta}_{1}, \delta_{2}, \delta_{3}$, and the perturbation maps $\phi, \theta, \rho$ are given in Table 5. (Note that the maps are defined in such a way that the squares in this system anti-commute.) The total complex of this system is then the following free $\mathbb{Z} G$-resolution of the integers:

$$
\begin{aligned}
& \ldots \stackrel{d_{4}}{\longrightarrow}(\mathbb{Z} G)^{9} \stackrel{d_{5}}{\longrightarrow}(\mathbb{Z} G)^{9} \stackrel{d_{4}}{\longrightarrow}(\mathbb{Z} G)^{9} \stackrel{d_{5}}{\longrightarrow}(\mathbb{Z} G)^{9} \\
& \quad \stackrel{d_{4}}{\longrightarrow}(\mathbb{Z} G)^{10} \stackrel{d_{3}}{\longrightarrow}(\mathbb{Z} G)^{13} \stackrel{d_{2}}{\longrightarrow}(\mathbb{Z} G)^{13} \stackrel{d_{1}}{\longrightarrow}(\mathbb{Z} G)^{6}
\end{aligned}
$$




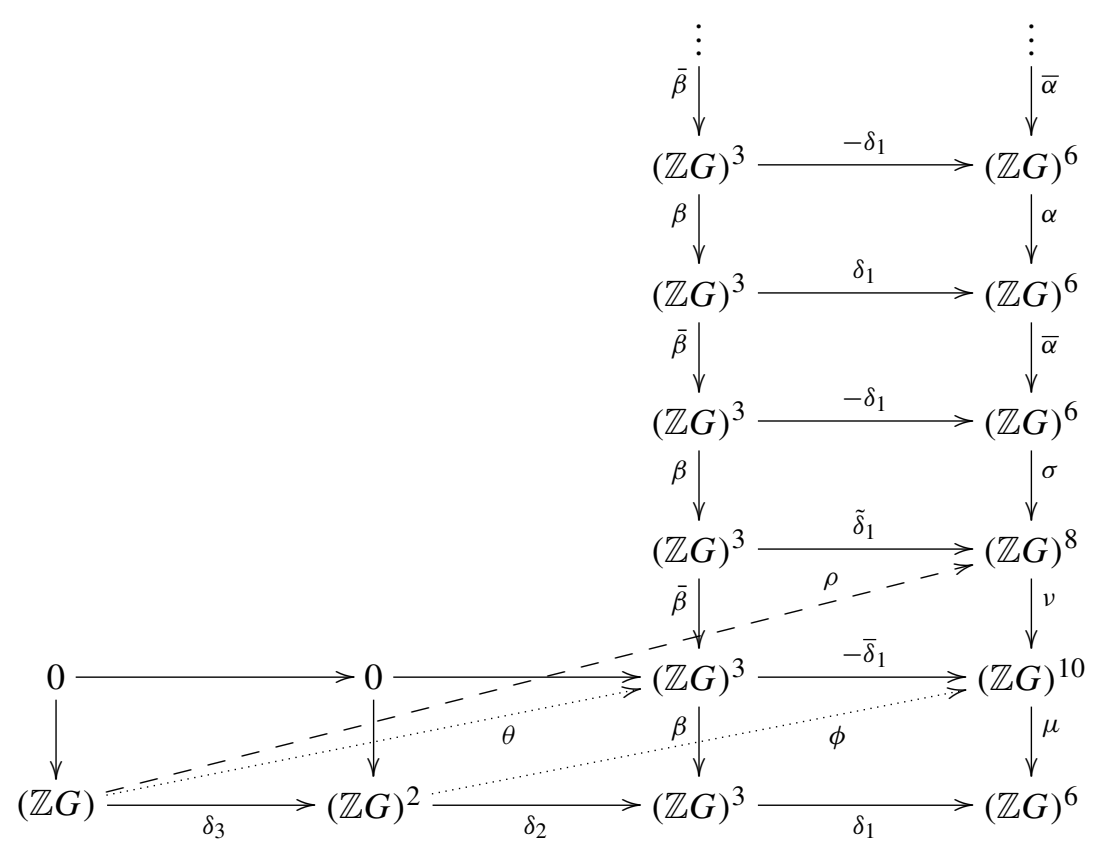

Figure 5. System of free $\mathbb{Z} G$-modules.

where $d_{1}, d_{2}, d_{3}, d_{4}, d_{5}$ are given by

$$
\begin{aligned}
& d_{1}=\mu+\delta_{1}, \\
& d_{2}=v-\bar{\delta}_{1}+\beta+\phi+\delta_{2}, \\
& d_{3}=\sigma+\tilde{\delta}_{1}+\bar{\beta}+\rho+\theta+\delta_{3}, \\
& d_{4}=\bar{\alpha}-\delta_{1}+\beta, \\
& d_{5}=\alpha+\delta_{1}+\bar{\beta} .
\end{aligned}
$$

Corollary 4. Let $G=G(l, m, n)$ where $2 /|l|+1 /|n| \leq 1$ and $2 /|m|+1 /|n| \leq 1$.

\section{Then}

$$
H_{k}(G, \mathbb{Z})= \begin{cases}\mathbb{Z} & k=0 \\ \mathbb{Z}_{l} \oplus \mathbb{Z}_{m} & k=1 \\ \mathbb{Z} & k=2 \\ \mathbb{Z}_{l} \oplus \mathbb{Z}_{m} \oplus \mathbb{Z}_{n} & k \geq 3, k \text { odd } \\ 0 & k \geq 4, k \text { even }\end{cases}
$$


Table 5. Boundary maps for system of free $\mathbb{Z} G$-modules.

\begin{tabular}{|c|c|}
\hline Summand & Boundary map \\
\hline$F_{3,0}$ & $\begin{array}{l}\delta_{3}\left(f^{3,0}\right)=\left(x^{-1}-1\right) f_{1}^{2,0}+\left(y^{-1}-1\right) f_{2}^{2,0} \\
\theta\left(f^{3,0}\right)=-x^{-1} f_{1}^{1,1}-y^{-1} f_{2}^{1,1}+y^{-1} x^{-1} f_{3}^{1,1} \\
\rho\left(f^{3,0}\right)=y^{-1} f_{1}^{0,2}+x^{-1} f_{5}^{0,2}\end{array}$ \\
\hline$F_{2,0}$ & $\begin{array}{l}\delta_{2}\left(f_{1}^{2,0}\right)=-f_{1}^{1,0}+\left(1-y^{-1}\right) f_{3}^{1,0} \\
\delta_{2}\left(f_{2}^{2,0}\right)=-f_{2}^{1,0}+\left(x^{-1}-1\right) f_{3}^{1,0} \\
\phi\left(f_{1}^{2,0}\right)=-f_{1}^{0,1}-y^{-1} f_{2}^{0,1}+\left(1-y^{-1}\right) f_{6}^{0,1}-y^{-1} f_{8}^{0,1} \\
\phi\left(f_{2}^{2,0}\right)=\left(1-x^{-1}\right) f_{1}^{0,1}-x^{-1} f_{3}^{0,1}-f_{6}^{0,1}-x^{-1} f_{7}^{0,1}\end{array}$ \\
\hline $\begin{array}{l}F_{1,2 q+1} \\
(q \geq 0)\end{array}$ & $\begin{array}{l}\beta\left(f_{1}^{1,2 q+1}\right)=(x-1) f_{1}^{1,2 q} \\
\beta\left(f_{2}^{1,2 q+1}\right)=(y-1) f_{2}^{1,2 q} \\
\beta\left(f_{3}^{1,2 q+1}\right)=([x, y]-1) f_{3}^{1,2 q}\end{array}$ \\
\hline $\begin{array}{l}F_{1,2 q} \\
(q \geq 1)\end{array}$ & $\begin{array}{l}\bar{\beta}\left(f_{1}^{1,2 q}\right)=\sum_{i=0}^{l-1} x^{i} f_{1}^{1,2 q-1} \\
\bar{\beta}\left(f_{2}^{1,2 q}\right)=\sum_{i=0}^{m-1} y^{i} f_{2}^{1,2 q-1} \\
\bar{\beta}\left(f_{3}^{1,2 q}\right)=\sum_{i=0}^{n-1}[x, y]^{i} f_{3}^{1,2 q-1}\end{array}$ \\
\hline $\begin{array}{l}F_{1, q} \\
(q \neq 1,2)\end{array}$ & $\begin{array}{l}\delta_{1}\left(f_{1}^{1, q}\right)=-f_{1}^{0, q}+y^{-1} f_{2}^{0, q} \\
\delta_{1}\left(f_{2}^{1, q}\right)=-f_{4}^{0, q}+x^{-1} f_{5}^{0, q} \\
\delta_{1}\left(f_{3}^{1, q}\right)=-f_{3}^{0, q}+f_{6}^{0, q}\end{array}$ \\
\hline$F_{1,2}$ & $\begin{array}{l}\tilde{\delta}_{1}\left(f_{1}^{1,2}\right)=-f_{2}^{0,2}+y^{-1} f_{3}^{0,2} \\
\tilde{\delta}_{1}\left(f_{2}^{1,2}\right)=-f_{6}^{0,2}+x^{-1} f_{7}^{0,2} \\
\tilde{\delta}_{1}\left(f_{3}^{1,2}\right)=-f_{4}^{0,2}+f_{8}^{0,2}\end{array}$ \\
\hline$F_{1,1}$ & $\begin{array}{l}\bar{\delta}_{1}\left(f_{1}^{1,1}\right)=-f_{3}^{0,1}+y^{-1} f_{4}^{0,1} \\
\bar{\delta}_{1}\left(f_{2}^{1,1}\right)=-f_{8}^{0,1}+x^{-1} f_{9}^{0,1} \\
\bar{\delta}_{1}\left(f_{3}^{1,1}\right)=-f_{5}^{0,1}+f_{10}^{0,1}\end{array}$ \\
\hline
\end{tabular}




\begin{tabular}{|c|c|}
\hline $\begin{array}{l}F_{0,2 q+1} \\
(q \geq 2)\end{array}$ & $\begin{array}{l}\alpha\left(f_{1}^{0,2 q+1}\right)=(x-1) f_{1}^{0,2 q} \\
\alpha\left(f_{2}^{0,2 q+1}\right)=\left(y x y^{-1}-1\right) f_{2}^{0,2 q} \\
\alpha\left(f_{3}^{0,2 q+1}\right)=([x, y]-1) f_{3}^{0,2 q} \\
\alpha\left(f_{4}^{0,2 q+1}\right)=(y-1) f_{4}^{0,2 q} \\
\alpha\left(f_{5}^{0,2 q+1}\right)=\left(x y x^{-1}-1\right) f_{5}^{0,2 q} \\
\alpha\left(f_{6}^{0,2 q+1}\right)=([x, y]-1) f_{6}^{0,2 q}\end{array}$ \\
\hline $\begin{array}{l}F_{0,2 q} \\
(q \geq 2)\end{array}$ & $\begin{array}{l}\bar{\alpha}\left(f_{1}^{0,2 q}\right)=\sum_{i=0}^{l-1} x^{i} f_{1}^{0,2 q-1} \\
\bar{\alpha}\left(f_{2}^{0,2 q}\right)=\sum_{i=0}^{l-1}\left(y x y^{-1}\right)^{i} f_{2}^{0,2 q-1} \\
\bar{\alpha}\left(f_{3}^{0,2 q}\right)=\sum_{i=0}^{n-1}[x, y]^{i} f_{3}^{0,2 q-1} \\
\bar{\alpha}\left(f_{4}^{0,2 q}\right)=\sum_{i=0}^{m-1} y^{i} f_{4}^{0,2 q-1} \\
\bar{\alpha}\left(f_{5}^{0,2 q}\right)=\sum_{i=0}^{m-1}\left(x y x^{-1}\right)^{i} f_{5}^{0,2 q-1} \\
\bar{\alpha}\left(f_{6}^{0,2 q}\right)=\sum_{i=0}^{n-1}[x, y]^{i} f_{6}^{0,2 q-1}\end{array}$ \\
\hline$F_{0,3}$ & $\begin{array}{l}\sigma\left(f_{1}^{0,3}\right)=(x-1) f_{2}^{0,2} \\
\sigma\left(f_{2}^{0,3}\right)=\left(y x y^{-1}-1\right) f_{3}^{0,2} \\
\sigma\left(f_{3}^{0,3}\right)=([x, y]-1) f_{4}^{0,2} \\
\sigma\left(f_{4}^{0,3}\right)=(y-1) f_{6}^{0,2} \\
\sigma\left(f_{5}^{0,3}\right)=\left(x y x^{-1}-1\right) f_{7}^{0,2} \\
\sigma\left(f_{6}^{0,3}\right)=([x, y]-1) f_{8}^{0,2}\end{array}$ \\
\hline$F_{0,2}$ & $\begin{aligned} v\left(f_{1}^{0,2}\right)= & \left(x^{-1}-1\right) f_{1}^{0,1}+\left(y x^{-1} y^{-1}-1\right) f_{2}^{0,1}+x^{-1} f_{3}^{0,1} \\
& \quad-y x^{-1} y^{-1} f_{4}^{0,1}-x^{-1} f_{5}^{0,1} \\
v\left(f_{2}^{0,2}\right)= & \sum_{i=0}^{l-1} x^{i} f_{3}^{0,1} \\
v\left(f_{3}^{0,2}\right)= & \sum_{i=0}^{l-1}\left(y x y^{-1}\right)^{i} f_{4}^{0,1} \\
v\left(f_{4}^{0,2}\right)= & \sum_{i=0}^{n-1}[x, y]^{i} f_{5}^{0,1} \\
v\left(f_{5}^{0,2}\right)= & \left(y^{-1}-1\right) f_{6}^{0,1}+\left(x y^{-1} x^{-1}-1\right) f_{7}^{0,1}+y^{-1} f_{8}^{0,1} \\
& \quad-x y^{-1} x^{-1} f_{9}^{0,1}+x y^{-1} x^{-1} f_{10}^{0,1}\end{aligned}$ \\
\hline
\end{tabular}




\begin{tabular}{|l|l|}
\hline & $v\left(f_{6}^{0,2}\right)=\sum_{i=0}^{m-1} y^{i} f_{8}^{0,1}$ \\
& $v\left(f_{7}^{0,2}\right)=\sum_{i=0}^{m-1}\left(x y x^{-1}\right)^{i} f_{9}^{0,1}$ \\
& $v\left(f_{8}^{0,2}\right)=\sum_{i=0}^{n-1}[x, y]^{i} f_{10}^{0,1}$ \\
\hline$F_{0,1}$ & $\mu\left(f_{1}^{0,1}\right)=f_{1}^{0,0}-f_{3}^{0,0}$ \\
$\mu\left(f_{2}^{0,1}\right)=-f_{2}^{0,0}+f_{3}^{0,0}$ \\
$\mu\left(f_{3}^{0,1}\right)=(x-1) f_{1}^{0,0}$ \\
$\mu\left(f_{4}^{0,1}\right)=\left(y x y^{-1}-1\right) f_{2}^{0,0} \mu\left(f_{5}^{0,1}\right)=([x, y]-1) f_{3}^{0,0}$ \\
$\mu\left(f_{6}^{0,1}\right)=f_{4}^{0,0}-f_{6}^{0,0}$ \\
$\mu\left(f_{7}^{0,1}\right)=-f_{5}^{0,0}+f_{6}^{0,0}$ \\
$\mu\left(f_{8}^{0,1}\right)=(y-1) f_{4}^{0,0}$ \\
$\mu\left(f_{9}^{0,1}\right)=\left(x y x^{-1}-1\right) f_{5}^{0,0}$ \\
$\mu\left(f_{10}^{0,1}\right)=([x, y]-1) f_{6}^{0,0}$
\end{tabular}

If, under the hypotheses of Theorem 3, we additionally have $|n| \geq 4$ then the homology groups $H_{k}(G, \mathbb{Z})(k \geq 3)$ can be obtained immediately from results of Howie (see Corollary D of [11], [12]). These results were obtained using algebraic techniques and they apply in the more general setting of one-relator products of groups.

For the remaining cases we provide only the form of a free $\mathbb{Z} G$-resolution over the integers. The method can of course be used to obtain the maps as well, if required.

Theorem 5. Let $G=G(l, m, n)$ where $2 /|l|+1 /|n|>1$ or $2 /|m|+1 /|n|>1$ and where $(\{|l|,|m|\},|n|) \neq(\{2,2\},|n|),(\{2,3\}, 2),(\{3,3\}, 2),(\{2,4\}, 2),(\{2,3\}, 3)$. Then there is a free $\mathbb{Z} G$-resolution of the integers

$$
\cdots \longrightarrow R_{6} \longrightarrow R_{5} \longrightarrow R_{4} \longrightarrow(\mathbb{Z} G)^{11} \longrightarrow(\mathbb{Z} G)^{12} \longrightarrow(\mathbb{Z} G)^{10} \longrightarrow(\mathbb{Z} G)^{4}
$$

where for $p \geq 4, R_{p}=(\mathbb{Z} G)^{p+7}$.

Proof. Without loss of generality, suppose $2 /|l|+1 /|n|>1$. Then $(|l|,|n|)=(2,|n|)$ or $(3,2)$ and $2 /|m|+1 /|n| \leq 1$. As described in the proof of Theorem 3 there is a faithful action of $G$ on $H^{3}$. In these cases $v_{1}, y^{-1} v_{1}$ are ordinary points in $H^{3}$ and the groups $\left\langle x, y x y^{-1}\right\rangle,\left\langle x, y x y^{-1}\right\rangle^{y}$ are isomorphic to the finite triangle 
group $T=T(l, l, n)$. Note that $T$ is either the dihedral group $D_{|2 n|}$ or the alternating group $A_{4}$. The points $v_{2}, x^{-1} v_{2}$ are again infinite or ideal points and the groups $\left\langle y, x y x^{-1}\right\rangle,\left\langle y, x y x^{-1}\right\rangle^{x}$ are isomorphic to the infinite triangle group $T^{\prime}=T(m, m, n)$.

As explained in Section 3, there is a free $\mathbb{Z} T$-resolution of the integers of the form

$$
S_{*}: \quad \cdots \longrightarrow S_{5} \longrightarrow S_{4} \longrightarrow S_{3} \longrightarrow S_{2} \longrightarrow S_{1} \longrightarrow S_{0}
$$

where for $p \geq 0, S_{p}=(\mathbb{Z} T)^{p+1}$. Also, there is a free $\mathbb{Z} T^{\prime}$-resolution of the integers of the form

$$
S_{*}^{\prime}: \quad \cdots \longrightarrow S_{5}^{\prime} \longrightarrow S_{4}^{\prime} \longrightarrow S_{3}^{\prime} \longrightarrow\left(\mathbb{Z} T^{\prime}\right)^{4} \longrightarrow\left(\mathbb{Z} T^{\prime}\right)^{5} \longrightarrow\left(\mathbb{Z} T^{\prime}\right)^{3}
$$

where for $p \geq 3, S_{p}^{\prime}=\left(\mathbb{Z} T^{\prime}\right)^{3}$. Combining these resolutions we get the following free resolution of $C_{0}(X)$

$$
F_{0, *}: \quad \cdots \longrightarrow F_{0,5} \longrightarrow F_{0,4} \longrightarrow F_{0,3} \longrightarrow(\mathbb{Z} G)^{7} \longrightarrow(\mathbb{Z} G)^{7} \longrightarrow(\mathbb{Z} G)^{4}
$$

where for $p \geq 3, F_{0, p}=(\mathbb{Z} G)^{p+4}$. The free resolutions $F_{1, *}, F_{2, *}, F_{3, *}$ of $C_{1}(X)$, $C_{2}(X), C_{3}(X)$ are as given in the proof of Theorem 3. Combining these four free resolutions we obtain a system of free $\mathbb{Z} G$-modules whose total complex is of the required form.

In addition to the groups $G(l, m, n)$, there are other classes of generalized triangle groups which are known to admit faithful representations as groups of isometries of hyperbolic 3-space. The second author has shown [22] that (except in the cases $(\{|l|,|m|\},|n|)=(\{2,3\}, 2),(\{2,2\}, n))$ the groups

$$
\tilde{G}=\left\langle x, y \mid x^{l}=y^{m}=\left((x y)^{2}\left(x^{-1} y^{-1}\right)^{2}\right)^{n}=1\right\rangle
$$

admit such a representation. The method of proof follows [9] in explicitly constructing a fundamental domain. The stabilizer groups of the edges of this domain are cyclic groups and the stabilizer groups of the vertices are triangle groups. A free $\mathbb{Z} \tilde{G}$-resolution of the integers can therefore be constructed exactly as in Section 5 . More generally, Jones and Reid [13] consider a class of generalized triangle groups which arise as the fundamental groups of 3-dimensional orbifolds whose singular sets are obtained by adding an unknotting tunnel to a 2-bridge knot or link. They show that in most cases such a group has a faithful representation as a group of isometries of hyperbolic 3-space. It seems likely that our method can also be applied in this setting.

Acknowledgement. We would like to thank the referee for the careful reading of this paper. 


\section{References}

[1] G. Baumslag, J. W. Morgan, and P. B. Shalen, Generalized triangle groups. Math. Proc. Cambridge Philos. Soc. 102 (1) (1987), 25-31. Zbl 0626.20023 MR 0886432

[2] R. Brown and J. Huebschmann, Identities among relations. In Low-dimensional topology (Bangor, 1979), London Math. Soc. Lecture Note Ser. 48, Cambridge University Press, Cambridge 1982, 153-202. Zbl 0485.57001 MR 0662431

[3] H. S. M. Coxeter, The groups determined by the relations $S^{t}=T^{m}=\left(S^{-1} T^{-1} S T\right)^{p}=1$. Part I. Duke. Math. J. 2 (1) (1936), 61-73. JFM 62.0080.02

[4] G. Ellis, J. Harris, and E. Sköldberg, Polytopal resolutions for finite groups. J. Reine Angew. Math., to appear.

[5] B. Fine, J. Howie, and G. Rosenberger, Ree-Mendelsohn pairs in generalized triangle groups. Comm. Algebra 17 (2) (1989), 251-258. Zbl 0664.20016 MR 0978473

[6] B. Fine, F. Roehl, and G. Rosenberger, The Tits alternative for generalized triangle groups. In Groups-Korea '98 (Pusan), Walter de Gruyter, Berlin 2000, 95-131. Zbl 0953.20023 MR 1751090

[7] B. Fine, G. Rosenberger, and M. Stille, Euler characteristic for one-relator products of cyclics. Comm. Algebra 21 (12) (1993), 4353-4359. Zbl 0792.20025 MR 1242835

[8] The GAP Group. GAP - Groups, Algorithms, and Programming, Version 4.4, 2004. (http: / /www.gap-system.org).

[9] M. A. Hagelberg, Hyperbolic 3-dimensional orbifolds. In Topics in knot theory (Erzurum, 1992), NATO Adv. Sci. Inst. Ser. C Math. Phys. Sci. 399, Kluwer Academic Publishers, Dordrecht 1993, 259-268. Zbl 0832.57008 MR 1257913

[10] M. Hagelberg, C. Maclachlan, and G. Rosenberger, On discrete generalised triangle groups. Proc. Edinburgh Math. Soc. (2) 38 (3) (1995), 397-412. Zbl 0836.20070 MR 1357638

[11] J. Howie, The quotient of a free product of groups by a single high-powered relator. I: Pictures. Fifth and higher powers. Proc. Lond. Math. Soc. (3) 59 (3) (1989), 507-540. Zbl 0653.20030 MR 1014869

[12] J. Howie, The quotient of a free product of groups by a single high-powered relator. II: Fourth powers. Proc. Lond. Math. Soc. (3) 61 (1) (1990), 33-62. Zbl 0705.20028 MR 1051098

[13] K. N. Jones and A. W. Reid, Minimal index torsion-free subgroups of Kleinian groups. Math. Ann. 310 (2) (1998), 235-250. Zbl 0890.57016 MR 1602004

[14] F. Levin and G. Rosenberger, A class of SQ-universal groups. In Group Theory ((Singapore, 1987), Walter de Gruyter, Berlin 1989, 409-415. Zbl 0659.20025 MR 0981859

[15] C. Maclachlan and G. J. Martin, The non-compact arithmetic generalised triangle groups. Topology 40 (5) (2001), 927-944. Zbl 1052.30043 MR 1860535

[16] C. Maclachlan and G. J. Martin, All Kleinian groups with two elliptic generators whose commutator is elliptic. Math. Proc. Cambridge Philos. Soc. 135 (3) (2003), 413-420. Zbl 1048.20034 MR 2018256

[17] S. J. Pride, Identities among relations of group presentations. In Group theory from a geometrical viewpoint (Trieste, 1990), World Sci. Publishing, River Edge, NJ, 1991, 687-717. Zbl 0843.20026 MR 1170381 
Vol. 80 (2005)

[18] A. Sinkov, The groups determined by the relations $S^{t}=T^{m}=\left(S^{-1} T^{-1} S T\right)^{p}=1$. Part II. Duke. Math. J. 2 (1) (1936), 74-83. JFM 62.0080.01

[19] R. Strebel, Explicit resolutions for the binary polyhedral groups and for other central extensions of the triangle groups. Comment. Math. Helv. 58 (3) (1983), 433-452. Zbl 0541.20035 MR 0727712

[20] È. B. Vinberg, J. Mennicke, and H. Helling, On some generalized triangular groups and three-dimensional orbifolds. Trans. Moscow Math. Soc. 56 (1995), 1-21. Zbl 0867.22009 MR 1468463

[21] C. T. C. Wall, Resolutions for extensions of groups. Proc. Cambridge Philos. Soc. 57 (1961), 251-255. Zbl 0106.24903 MR 0178046

[22] A. G. T. Williams, Studies on generalised triangle groups. Ph.D. thesis, Heriot Watt University, 2000.

[23] A. G. T. Williams, Euler characteristics for orbifold generalized triangle groups. Math. Proc. Cambridge Philos. Soc. 132 (3) (2002), 435-438. Zbl 1025.20021 MR 1891681

Received June 10, 2004

Graham Ellis, Department of Mathematics, National University of Ireland, Galway, Ireland

E-mail: graham.ellis@ nuigalway.ie

Gerald Williams, Institute of Mathematics, Statistics, and Actuarial Science, University of Kent, Canterbury CT2 7NF, U.K.

E-mail: g.williams@kent.ac.uk 